\title{
Development Model of Business Management Based on Big Data
}

\author{
Zhongtian $\mathrm{Xie}^{1 *}$ \\ ${ }^{1}$ China University of Petroleum,Beijing
}

\begin{abstract}
In recent years, with the continuous development of network information technology, a large amount of data information has gradually appeared in real life. One of the most obvious examples is the new data management development model based on big data. In the context of big data, e-commerce has developed rapidly, and products and services have become more diverse. However, at the same time, there are many problems, such as insecurity of user information, increasing differences in transactions, and lack of management strategies. This article mainly introduces how to solve existing problems and make business management more suitable for the arrival of the big data era. This article uses bibliographic analysis, benchmark analysis, graphical analysis and case analysis to conduct an in-depth discussion on theoretical research and commercial applications of big data. Classify and divide ubiquitous different types and irregular massive data, and propose different big data solutions according to different categories. Finally, a complete set of big data management model was established to verify the feasibility of the big data management model. Big data has increased the efficiency of business management by nearly $30 \%$.
\end{abstract}

\section{Introduction}

\subsection{Background and Significance}

Since entering the 21 st century, the new technological revolution has entered a period of rapid development. The Internet, the Internet of Things, cloud computing and other technologies have achieved rapid growth, the proliferation of mobile devices, the rise of social networks, and ecommerce and other communication methods have been widely used and spread. These interconnections have caused an explosive growth in the amount of global data, and the growth rate is higher than any previous period [1]. As a result, in recent years, the concept of "big data" has been proposed and has received widespread attention from the country, researchers, companies and society. The concept of big data includes not only massive data, but also related data processing and analysis techniques [2]. China's market economy environment has increased the vitality of enterprises and expanded their independent management rights. Some companies have achieved rapid growth through innovative business models, and companies have begun to consider choosing business models and choosing business models in the process of continuous growth and development. The impact of innovation on its own development [3-4].

The development of big data has many impacts on social life. This paper systematically discussed the development of big data, and carried out specific analysis and research on the influence and innovation of business models based on big data [5]. Summarize the scope of big data [6], and make further explanations and expansions based on your own research based on the previous research, hoping to provide support for other researchers in the field of further research [7].

\subsection{Related Work}

Due to the importance of big data, more and more scholars and research teams have begun to study big data and have achieved good results [8]. For example: Rathore once studied business development models from short-term gains[9]. This method has a very high short-term effect. However, because of the rapid development of the social Internet leading to long-term results, his prediction results are not accurate. not tall. Therefore, this paper proposes a business management development model based on big data to judge whether big data plays a positive role in the development of business management [10].

\subsection{Main Content}

In order to solve the problem of business management development, this article uses literature analysis, comparative analysis, graphical analysis and case analysis to conduct an in-depth discussion on the theoretical research and commercial application of big data. The prospect of the management development model, summarizes the business management development method based on big data, through the method of this article, the efficiency rate of big data for business management has increased by nearly $30 \%$.

*xiezhongtian0727@163.com 


\section{Research Method of Business Management Development Model Based on Big Data}

\subsection{Literature Research Method and Data Analysis Method}

By consulting domestic and foreign journals and articles, consulting big data theory databases and industry analysis reports, we can understand the main directions and topics of external big data research. Check the technical documentation of the relevant big data provider and the consulting analysis report of the consulting service to understand the description and design concept of the relevant product. Improve your big data knowledge system by consulting business books and documents related to big data.

\subsection{Comparative Analysis}

In the analysis, this article will analyze the differences between traditional applications and business management models based on big data systems, use comparative analysis to discover the differences between the two, help understand the advantages and characteristics of big data, and form a large data system The difference between business management framework model and traditional framework model

\subsection{Case Analysis Method}

This article will take the business process of an ecommerce platform as an example to show the problems and model practices that need to be paid attention to when implementing the solutions proposed in this article in a business environment.

\subsection{Graphical Analysis Method}

Since applications and applications include extensive knowledge in the fields of software engineering and business management, in order to express the ideas of this article intuitively, this article will introduce some graphics and models to intuitively understand the problems and solutions discussed in this article. .

\section{Case Comparison Experiment of Business Management Development Model Based on Big Data}

\subsection{Online Loan Application Process and Decision-Making Process}

Take Taobao credit loan as an example. Taobao credit loan application procedure is very convenient, from loan to repayment is completed online. After submitting the client application, Ali will collect the loan applicant's previous operating data, cash flow, credit certificate and other information for analysis, and decide whether to arrange overseas visits and video surveys as needed. If necessary, Ali instructs a third party to conduct on-site inspections of the company, meet with the applicant to understand the company's business development, collect relevant information required for loans, take pictures of relevant information, and determine whether it is compatible. Contact relevant departments for further investigation. The video survey was conducted by Alibaba Financial Corporation itself. After completing the above steps, all the applicant has to do is to wait for the approval result. One thing that needs to be explained is that Alibaba's small loans may not get as many loans as the loan applicant's customers, but based on the loan applicant's operation and credit analysis, the loan application manager will receive the application. One of the loan amounts available to loan customers is online. Only the amount within the amount applied by the loan applicant can be fully satisfied, otherwise the loan can only reach the amount at most. According to the collected data analysis, the number of people participating in online loans from 2015 to 2019 was 43 million, 45 million, 48 million, 50 million and 60 million.

\subsection{Application Process and Decision-Making Process of Traditional Bank Loans}

Traditional bank loans refer to an economic behavior in which the bank lends funds to people who need interest at a specific interest rate in accordance with national policies and agrees to repay it on time. Applying for a home mortgage loan online is not easy. Applicants must apply at the bank office. The bank loan manager will first receive a preliminary judgment on the applicant's materials. If the submitted materials do not meet the requirements, it is very likely that the materials will not be filled in. If you want to complete the materials and procedures, the applicant must go to the relevant agency to issue the corresponding certificate. Cumbersome procedures may not work. After submitting the information, the bank staff should also go to the applicant's company or institution to conduct an on-site investigation, take a photo and leave a certificate, fill in the investigation report and wait for the meeting result to pass. Only after the meeting is approved, the system can submit approval to receive the requested loan. According to the collected data, the number of people participating in traditional bank loans from 2015 to 2019 was 20 million, 34 million, 38 million, 40 million and 410 million.

\section{Comparative Analysis of Business Management Development Models Based on Big Data}

\subsection{Comparative Results of Ali Small Loans and Traditional Bank Loans}

From the above experiment, the Ali microloans and traditional bank loans are analyzed from three aspects: guarantee requirements, main target customers, and the number of participants in 2015-2020, as shown in Table 1. 
Table1. Comparison of Alibaba's small loans and traditional bank loans

\begin{tabular}{|c|c|c|}
\hline Loan category & Ali small loan & $\begin{array}{c}\text { Traditional } \\
\text { bank loans }\end{array}$ \\
\hline Guarantee demand & NO & YES \\
\hline Main target customers & $\begin{array}{c}\text { small and } \\
\text { medium-sized } \\
\text { enterprises }\end{array}$ & $\begin{array}{c}\text { large } \\
\text { enterprises }\end{array}$ \\
\hline $\begin{array}{c}\text { Number of participants } \\
(2015-2019)\end{array}$ & $\begin{array}{c}4300,4500,48 \\
00,5000,6000\end{array}$ & $\begin{array}{c}2000,3400,380 \\
0,4000,4100\end{array}$ \\
\hline
\end{tabular}

It can be seen from the comparison in the table that Ali microloans are more popular, more convenient and faster than traditional banks, and are welcomed by the public. These not only reflect the huge impact of big data on the business model innovation of the financial industry, but also reflect the unlimited potential and feasibility of big data and its technology in the business model. With the continuous development of Internet + , more and more people are using the Internet to complete transactions and innovation, and believe that more and more data creation will promote the continuous development of the Internet credit industry.

\subsection{Analyze Big Data Elements to Innovate Business Management Models}

The essence of business model innovation is to rethink the value creation model. A comparative analysis of Alibaba's microfinance and traditional bank loans shows that Alibaba's big data-driven microfinance innovation business model not only solves the financial difficulties of small and micro enterprises, but also promotes the development and improvement of commercial bank credit. Alibaba microfinance is an innovation in the business model of big data-owned companies. The business model innovation of large data-driven companies refers to companies with large amounts of user information, such as Google, Taobao, Facebook and other Internet companies. On the one hand, they can change the traditional marketing model through big data analysis of user information to achieve accurate marketing recommendations and personalized advertising; on the other hand, they can also provide other companies with their own data analysis big data solutions You can get other resources you need. According to the number of participants in Ali small loans and traditional bank loans from 2015 to 2019, the trend of big data applications can be predicted, as shown in Figure 1:

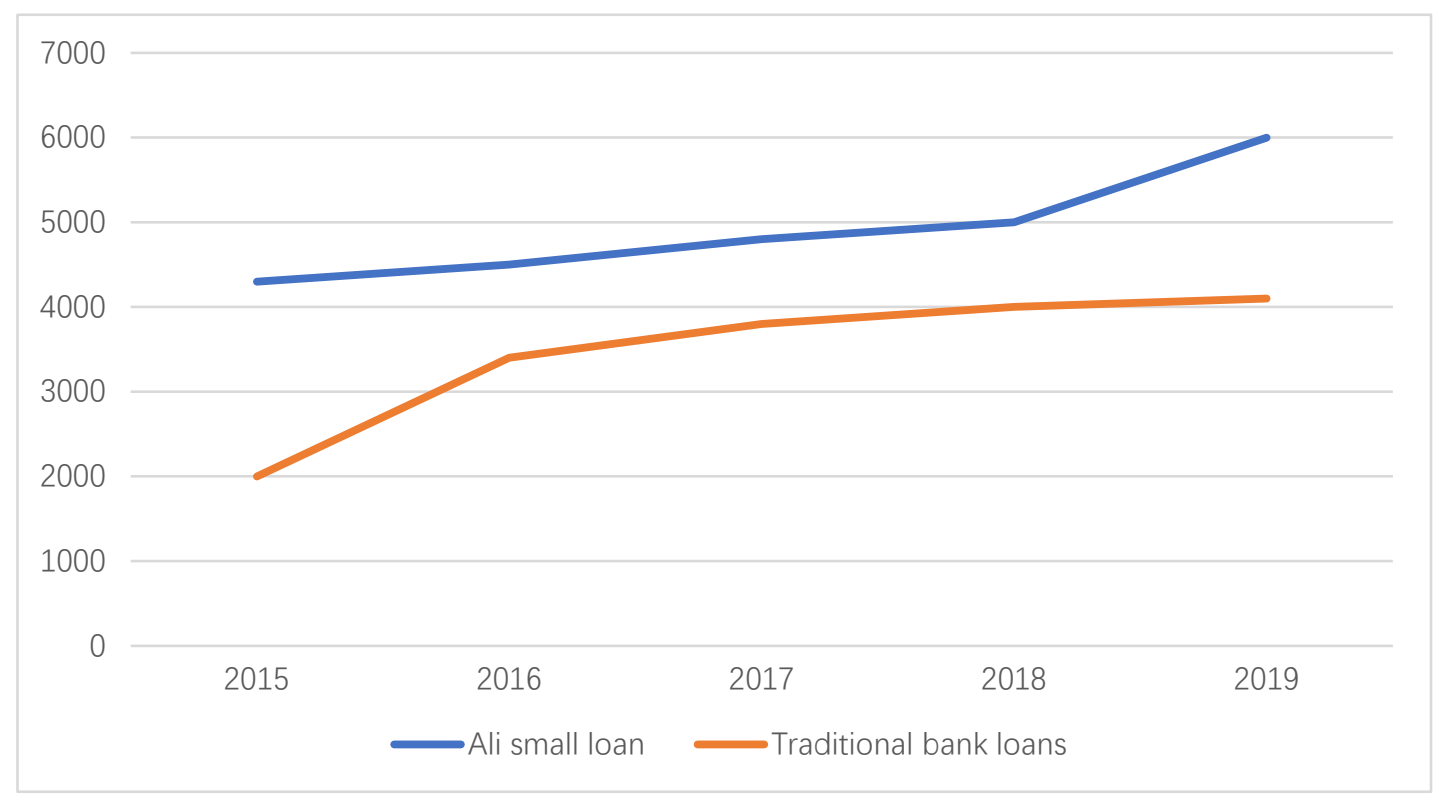

Figure 1. 2015-2019 Trends in the number of participants in Ali small loans and traditional bank loans

Alibaba microfinance is based on the credibility of big data. According to the loan application requirements, Alibaba will review and approve the loan application requirements of loan applicants through the ranking of its previous transaction and credit data and technology. Alibaba cloud computing and video. According to the loan needs of different applicants, the credit limit is determined through the quantitative model developed by Ali. Within the specified credit limit, applicants can flexibly choose preferred loan methods and repayment methods to a certain extent. And because the applicant's sales and other data are constantly changing, Alibaba microfinance can quickly respond to the applicant's credit based on "custom" data and adjust it according to the situation, even for large loans. "The value proposition of loan application service innovation can be realized, which is impossible for traditional credit institutions. Alibaba Microfinance is committed to providing services to small, medium and small enterprises and sellers within Alibaba, and has introduced credit Philosophy. The product "trades small for beauty" has achieved good growth in practice. 
The emergence of Alibaba's microfinance is the result of big data value discovery in big data analysis and extraction and financial business model innovation. The continuous advancement of technology can improve related algorithms and models to reduce the possibility of non-performing loans. On the other hand, through data analysis, it can also discover the growth trend of the credit market and adjust its credit products or launch new credit products in time. Meet the ever-changing market needs.

\section{Conclusions}

Because big data has completely subverted the industry, it is destined to have high commercial value and transformative power in the business management model, so it has broad application prospects. Big data will lead to the reconstruction of the company's form. It can provide users with resources, data and applications as services over the Internet. At the same time, big data is also a new method of infrastructure management that can transform a large number of highly virtualized resources. Manage resources to form a huge resource set and provide services equally. However, this article still has the following shortcomings: (1) Regional e-commerce research is a relatively new theory, and its own research is not perfect, so the analysis of regional e-commerce development evaluation system may not be complete. And go deep. (2) The elasticity index was used as one of the evaluation indexes for the first time. There may be some inaccurate and incomplete data collection and organization. With the huge expansion of data, how to use network data to reasonably reflect the actual development of the industry is still in the research stage, so the research in this article is also a preliminary exploration. (3) When proposing a regional e-commerce development strategy, it expressed constructive opinions on the establishment of a regional third-party e-commerce network platform, but due to the infinite nature of the Internet, this limited the geographic area of the platform.

\section{References}

1. Scott S L, Blocker A W, Bonassi F V, et al. Bayes and big data: the consensus Monte Carlo algorithm[J]. International Journal of Management ence \& Engineering Management, 2016,9(4):78-88.

2. Wang, Gang, Gunasekaran, et al. Big data analytics in logistics and supply chain management: Certain investigations for research and applications.[J]. International Journal of Production Economics, 2016,10(5):56-67.

3. Baccarelli E, Cordeschi N, Mei A, et al. Energyefficient dynamic traffic offloading and reconfiguration of networked data centers for big data stream mobile computing: review, challenges, and a case study[J]. Computers \& Chemical Engineering, 2016, 91(2):182-194.

4. Mei, Alessandro, Baccarelli, et al. Energy-Efficient Dynamic Traffic Offloading and Reconfiguration of Networked Data Centers for Big Data Stream Mobile
Computing: Review, Challenges, and a Case Study[J]. IEEE Network the Magazine of Computer Communications, 2016, 30(5):134-190.

5. Chen M, Ma Y, Song J , et al. Smart Clothing: Connecting Human with Clouds and Big Data for Sustainable Health Monitoring[J]. Mobile Networks \& Applications, 2016, 21(5):1-21.

6. Lei Y, Jia F, Lin J, et al. An Intelligent Fault Diagnosis Method Using Unsupervised Feature Learning Towards Mechanical Big Data[J]. IEEE Transactions on Industrial Electronics, 2016, 63(5):3137-3147.

7. He X, Ai Q, Qiu R C, et al. A Big Data Architecture Design for Smart Grids Based on Random Matrix Theory[J]. IEEE Transactions on Smart Grid, 2017, 8(2):674-686.

8. Wang Y, Kung L A, Byrd T A . Big data analytics: Understanding its capabilities and potential benefits for healthcare organizations[J]. Technological Forecasting \& Social Change, 2018, 126(JAN.):3-13.

9. Rathore M M , Ahmad A, Paul A, et al. Urban planning and building smart cities based on the Internet of Things using Big Data analytics[J]. Computer Networks the International Journal of Computer \& Telecommunications Networking, 2016, 101(C):63-80.

10. Scott S L, Blocker A W, Bonassi F V, et al. Bayes and big data: the consensus Monte Carlo algorithm[J]. International Journal of Management ence \& Engineering Management, 2016,97(8):78-88. 